\title{
Progesterone receptor membrane component 1 expression and putative function in bovine oocyte maturation, fertilization, and early embryonic development
}

\author{
Alberto M Luciano ${ }^{1}$, Valentina Lodde ${ }^{1,3}$, Federica Franciosi ${ }^{1}$, Fabrizio Ceciliani ${ }^{2}$ \\ and John J Peluso ${ }^{3}$ \\ ${ }^{1}$ Division of Veterinary Anatomy and Histology, Department of Animal Sciences and ${ }^{2}$ Department of Animal \\ Pathology and Veterinary Public Health, Faculty of Veterinary Medicine, University of Milan, Via Celoria 10, \\ 20133 Milan, Italy and ${ }^{3}$ Department of Cell Biology, University of Connecticut Health Center, Farmington, \\ Connecticut 06030, USA
}

Correspondence should be addressed to A M Luciano; Email: alberto.luciano@unimi.it

\begin{abstract}
Although the mRNA that encodes progesterone receptor membrane component 1 (PGRMC1) is present in mammalian oocytes, nothing is known about either PGRMC1's expression pattern or function in oocytes during maturation, fertilization, and subsequent embryonic development. As PGRMC1 associates with the mitotic spindle in somatic cells, we hypothesized that PGRMC1 is involved in oocyte maturation (meiosis). Western blot analysis confirmed the presence of PGRMC1 in bovine oocytes. This study also shows that PGRMC1 is present at the germinal vesicle (GV)- and MII-stage oocytes and is associated with male and female pronucleus formation of the zygote and is highly expressed in blastocysts. A more detailed examination of PGRMC1 localization using confocal imaging demonstrated that in GV-stage oocytes, PGRMC1 was concentrated throughout the GV but did not localize to the chromatin. With the resumption of meiosis in vitro, PGRMC1 concentrated in the centromeric region of metaphase I chromosomes, while in the anaphase I/telophase I stages the majority of PGRMC1 concentrated between the separating chromosomes. At the metaphase II stage, PGRMC1 re-associated with the centromeric region of the chromosomes. A colocalization study demonstrated that PGRMC1 associated with the phosphorylated form of aurora kinase B, which localizes to the centromeres at metaphase. Finally, PGRMC1 antibody injection significantly lowered the percentage of oocytes that matured and reached the metaphase II stage after $\mathbf{2 4} \mathrm{h}$ of culture. The majority of the PGRMC1 antibodyinjected oocytes arrested in the prometaphase I stage of meiosis. Furthermore, in most of the PGRMC1 antibody-injected oocytes, the chromosomes were disorganized and scattered. Taken together, these data demonstrate that PGRMC1 is expressed in bovine oocytes and its localization changes at specific stages of oocyte maturation. These observations suggest an important role for PGRMC1 in oocyte maturation, which may be specifically related to the mechanism by which chromosomes segregate.

Reproduction (2010) $140663-672$
\end{abstract}

\section{Introduction}

Progesterone receptor membrane component 1 (PGRMC1) is an evolutionary conserved protein, which is involved in regulating numerous biological functions (Cahill 2007). Recently, PGRMC1 was detected among the proteins of the mitotic spindle (Nousiainen et al. 2006), suggesting a role for it in mitosis. Interestingly, PGRMC1 is also expressed in bovine (Dode et al. 2006), rat (Peluso et al. 2006), and human oocytes (Wood et al. 2007). Moreover, the mRNA that encodes PGRMC1 is present in bovine oocytes at a very high level similar to that of actin (Dode et al. 2006). In spite of its high level of expression, little is known about PGRMC1 and oocyte function. Since PGRMC1 is present in mitotic spindle
(Nousiainen et al. 2006), it could be directly involved in regulating oocyte maturation (meiosis).

In mammalian ovaries, oocytes are arrested in prophase of the first meiotic division (i.e. germinal vesicle $(\mathrm{GV})$ stage) until the gonadotropin surge induces meiosis in oocytes of preovulatory follicles. Nuclear meiotic maturation of oocytes includes condensation of chromosomes and the sequential progression through prometaphase I, metaphase I, anaphase I, and telophase I. The meiotic progression then arrests at metaphase II (Eppig et al. 2004). This sequence of meiotic events also occurs in vitro. For example in cows, fully grown oocytes extracted from follicles larger than $2 \mathrm{~mm}$ are capable of resuming meiosis in vitro with $\sim 90 \%$ of the oocytes reaching metaphase II (Sirard et al. 1988). Regardless of 
whether meiosis occurs in vivo or in vitro, this process requires the precise coordination between the chromosomes and the tubulin-based meiotic spindle.

To guarantee the coordinated interaction between the chromosomes and the meiotic spindle, the activity and localization of various spindle-associated proteins is strictly regulated (Musacchio \& Salmon 2007). One important spindle-associated protein is aurora kinase $B$ (AURKB). AURKB is a protein kinase that is a component of the chromosomal passenger complex (CPC), which also consists of an inner centromere protein, INCENP, which binds AURKB (Ruchaud et al. 2007). During mitosis, AURKB plays a critical role in chromosomemicrotubule interactions (Hauf et al. 2003). This kinase is localized to the kinetochores from prophase to metaphase and relocates to the central spindle and the midbody in cytokinesis (Carmena \& Earnshaw 2003). The altered expression AURKB is implicated in defects of centrosome function, spindle assembly, and chromosomal instability (Ota et al. 2002, Fu et al. 2007).

AURKB is also expressed in mammalian oocytes (Jelinkova \& Kubelka 2006, Swain et al. 2008, Uzbekova et al. 2008, Shuda et al. 2009). It gradually becomes phosphorylated on threonine 232 (Thr232) during oocyte maturation. Phosphorylation of AURKB activates this kinase and leads to the phosphorylation of histone $\mathrm{H} 3$ and the gradual condensation of chromatin (Jelinkova \& Kubelka 2006, Wang et al. 2006, Swain et al. 2008). Moreover, altered AURKB activity increases the risk in chromosome congression failure, nondysjunction, and aneuploidy in mammalian oocytes (Vogt et al. 2009).

Since PGRMC1 is highly expressed in bovine oocytes and zygotes (Dode et al. 2006) and it is localized to the mitotic spindle (Nousiainen et al. 2006), we hypothesized that PGRMC1 is involved in meiosis. This study was designed to determine the role of PGRMC1 in oocyte meiotic division by monitoring its i) localization during oocyte in vitro maturation, IVF, and early development; ii) relationship to changes in the activity of AURKB; and iii) function by injecting an antibody to PGRMC1 into oocyte before in vitro maturation.

\section{Results}

\section{PGRMC1 expression in oocytes, zygotes, and embryos}

As shown in Fig. 1, western blot analysis demonstrated that PGRMC1 was specifically detected in bovine oocytes as a $\approx 26 \mathrm{kDa}$ protein. Having established that this antibody specifically detects bovine PGRMC1, it was utilized to localize PGRMC1 in bovine oocyte undergoing meiosis in vitro. Note that under culture conditions used in this study, GV-stage oocytes resume meiosis with $93 \%$ of the oocytes reaching metaphase II by $24 \mathrm{~h}$ of culture (Table 1 ). The rate of IVF and blastocyst development was respectively 89.5 and $37.3 \%$. In a preliminary study, PGRMC1 was localized at the time

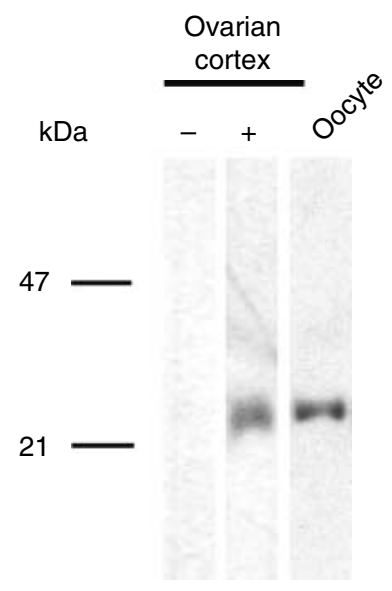

Figure 1 Detection of PGRMC1 in bovine oocytes. Western blot analysis showing PGRMC1 expression in bovine oocytes. A western blot using lysate prepared from bovine ovarian cortex, conducted in the absence of the PGRMC1 antibody, is shown as a negative control $(-)$ while a western blot using this lysate conducted in the presence of the PGRMC1 antibody is shown as a positive control $(+)$. Western blot using lysate prepared from bovine oocytes is shown in lane 3.

Representative blots from three independent experiments are shown.

of collection or after in vitro maturation, IVF, and embryo culture, and then observed in epifluorescence microscopy. As shown in Fig. 2, PGRMC1 was present within GV in immature oocytes and associated with the MII plate of in vitro matured oocytes. After IVF, PGRMC1 was associated with the female and the male pronucleus. At the blastocyst stage, virtually all the blastomeres showed positive staining for PGRMC1.

Having confirmed that PGRMC1 was present in bovine oocytes and embryos, changes in the localization of PGRMC1 during oocyte maturation was examined in more detail using confocal microscopy. This analysis revealed that PGRMC1 associates with the metaphase II chromosomes at distinct focal points (Supplementary Movie 1, see section on supplementary data given at the end of this article), which suggests that PGRMC1 localizes to the centromere.

As shown in Fig. 2, PGRMC1 was not detectable in the ooplasm. In fact, the fluorescent staining of the

Table 1 Sequential meiotic stages during bovine oocyte in vitro maturation

\begin{tabular}{lccccccc}
\hline $\begin{array}{l}\text { Time* } \\
(\mathrm{h})\end{array}$ & $\boldsymbol{n}$ & Pro I & Promet I & Met I & Ana I & Telo I & Met II \\
\hline 0 & 68 & $\mathbf{9 9}$ & 1 & & & & \\
4 & 76 & $\mathbf{6 4}$ & 36 & & & & \\
8 & 71 & 25 & $\mathbf{6 2}$ & 13 & & & \\
12 & 72 & 10 & 39 & $\mathbf{4 3}$ & 7 & 1 & \\
16 & 70 & & 6 & 30 & $\mathbf{3 7}$ & 17 & 10 \\
20 & 76 & 1 & 3 & 12 & 13 & $\mathbf{3 2}$ & 39 \\
24 & 72 & & & & 3 & 4 & $\mathbf{9 3}$
\end{tabular}

*Time in culture. Values in bold indicate the highest percentage of oocytes in specific stages of meiosis for each time in culture. Pro I, prophase I; Promet I, Prometaphase I; Met I, metaphase I; Ana I, anaphase I; Telo I, telophase I; Met II, metaphase II. 

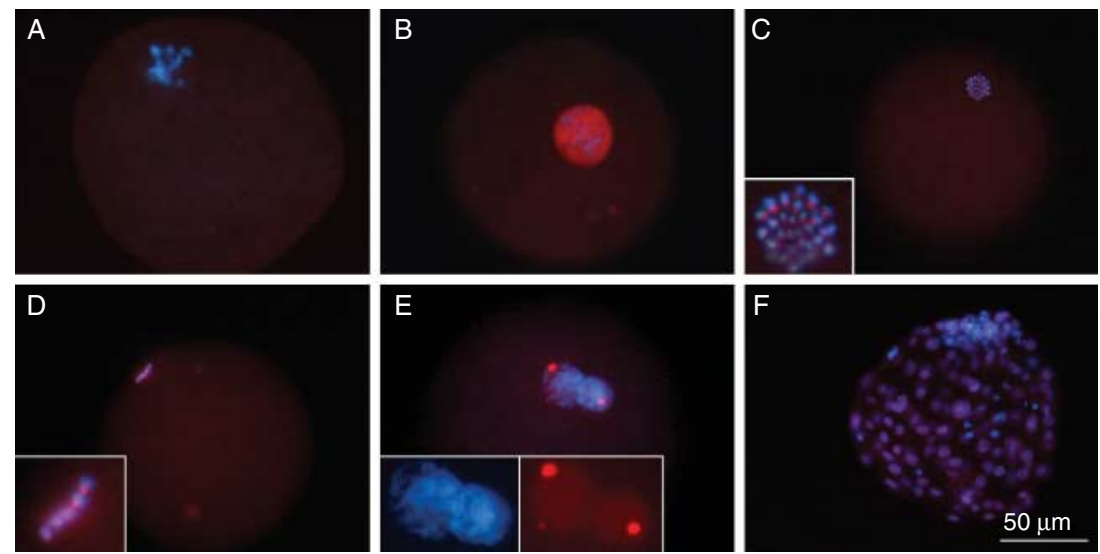

Figure 2 (A) A GV-stage negative control in which IgGs were used in place of the primary PGRMC1 antibody. PGRMC1 localization in prophase I (GV stage) (B), metaphase II oocyte (C, polar view and D, lateral view), pronuclear stage (E), and blastocyst-stage embryo (F). PGRMC1 (red) immunostaining is merged with DAPI (DNA; blue). Note that PGRMC1 appears to associate with germinal vesicle in immature oocyte and with the metaphase chromosomes in MII oocyte. In (C) and (D) the insets show the magnified metaphase II plate. In zygotes, PGRMC1 is associated with male and female pronucleus and is localized in the blastomeres up to the blastocyst stage of development. In (E) the insets show the magnified pronuclei; PGRMC1 and nuclear staining are shown separately to better illustrate pronuclear localization. Representative images in epifluorescence are shown. This immunocytochemical analysis was conducted on a total of 98 oocytes at the following stages: 39 in prophase I, 27 in metaphase II, 17 in pronuclear, and 15 in blastocyst.

cytoplasm in the IgG control was similar to that observed in the PGRMC1 antibody immunostained group (compare Fig. 2A and B).

\section{PGRMC1 expression in oocytes during meiosis in vitro and PGRMC1 and AURKB colocalization}

To test this assumption, PGRMC1 was colocalized with AURKB, which is known to associate with proteins that comprise the centromere at metaphase (Ruchaud et al. 2007). This study demonstrated that in prophase I (GV stage) oocytes, PGRMC1 and AURKB were highly expressed within the $\mathrm{GV}$, but neither protein was associated with the chromatin. At prometaphase I (i.e. after GV breakdown), both proteins began to associate with the chromosomes and this association was more evident in metaphase I oocytes. During anaphase I/ telophase I stages, both proteins dissociated from the chromosomes and concentrated in the mid-zone and the midbody of the separating chromosomal plates. At anaphase I, these two proteins colocalized throughout the mid-zone. During telophase, some AURKB colocalized to PGRMC1 at the center of the midbody between the chromosomal plates. Interestingly, some AURKB at the periphery of the midbody was not colocalized with PGRMC1. PGRMC1 and AURKB appeared to localize to the spindle pole in some oocytes. However, this was not consistently observed and its functional significance, if any, remains to be determined in further studies. Finally, at the metaphase II stage, both PGRMC1 and AURKB again colocalized to the centromeric region of the chromosomes (Fig. 3).

In a third study, the relationship between the AURKB and the Thr232 phosphorylated (active) form of AURKB was assessed. This study illustrated that AURKB began to associate with the chromatin in all of the prometaphase I oocytes, but the active form of AURKB was only barely detectable in about half of these oocytes. By metaphase I, staining for phosphorylated AURKB was readily detectable in all the oocytes and remained so up to the metaphase II stage (Fig. 4). Importantly, after GV breakdown, PGRMC1 was always concentrated to those subcellular compartments where the active, phosphorylated form of AURKB was present. This was particularly evident at the telophase I stage (compare Figs 3 and 4).

\section{PGRMC1 and oocyte maturation}

To begin to assess the role of PGRMC1 on oocyte maturation, GV-stage oocytes within their cumulus cell mass were injected with antibody to PGRMC1 while controls were injected with either IgG or PBS. After $24 \mathrm{~h}$, only $22 \%(n=68)$ of the oocytes injected with the PGRMC1 antibody progressed to metaphase II compared with $74 \%(n=39 ; P<0.05)$ and $68 \%(n=43 ; P<0.05)$ of PBS or IgG-injected oocytes respectively (Fig. 5A). A high proportion of PGRMC1 antibody-injected oocytes $(57.4 \%)$ arrested in prometaphase I stage. Some of the PGRMC1 antibody-injected oocytes progressed beyond prometaphase I as $5.8 \%(n=4), 8.8 \%(n=6)$, and $22 \%$ $(n=15)$ reached metaphase I, anaphase/telophase I, and metaphase II respectively, with most of these possessed chromosomes that were disorganized and scattered throughout the ooplasm (compare Fig. 5C with B).

\section{Discussion}

This study confirms and expands a previous mRNAbased observation (Dode et al. 2006) by demonstrating that PGRMC1 is present in bovine oocytes as a 

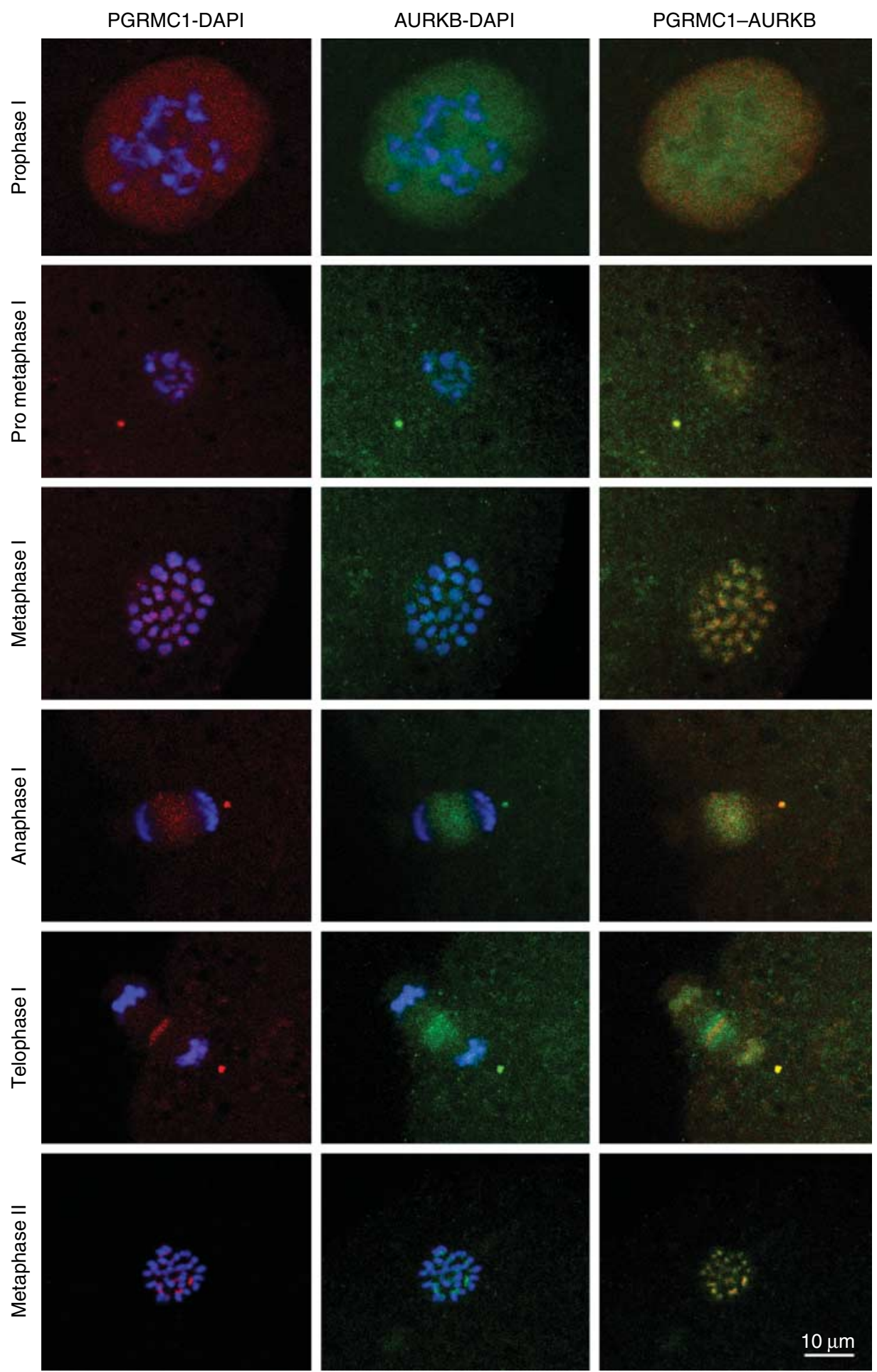

Figure 3 Colocalization of PGRMC1 and AURKB during bovine oocyte maturation. Representative confocal images showing PGRMC1 and AURKB localization at the indicated meiotic stages, from the prophase I (GV stage) to the metaphase II. Images were cropped to show the area containing the chromatin. PGRMC1 (red) and AURKB (green) immunostaining are merged with DAPI (DNA; blue) counterstained image to visualize the specific protein-chromosomes associations. In the right column, PGRMC1 and AURKB images are merged to reveal sites of their colocalization (orange). Note that the images of anaphase I and telophase I are in lateral view to better illustrate the relationship between PGRMC1 and AURKB at the midzone and the midbody. The images of metaphase I and metaphase II are shown in a polar view to better illustrate the relationship between PGRMC1, AURKB, and the chromosomes. This immunocytochemical analysis was conducted on a total of 199 oocytes at the following stages: 21 in prophase I, 17 in prometaphase I, 39 in metaphase I, 38 in anaphase I, 34 in telophase I, and 50 in metaphase II. 

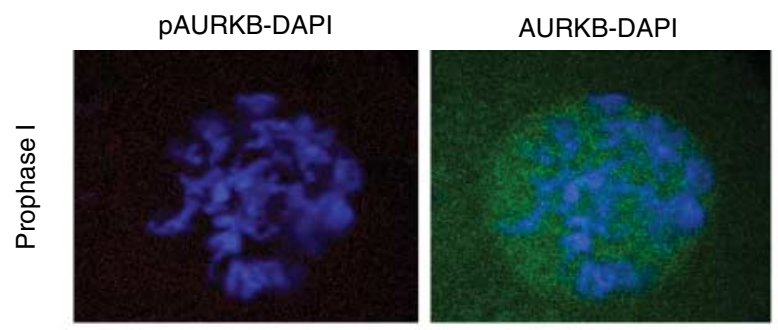

PAURKB-AURKB
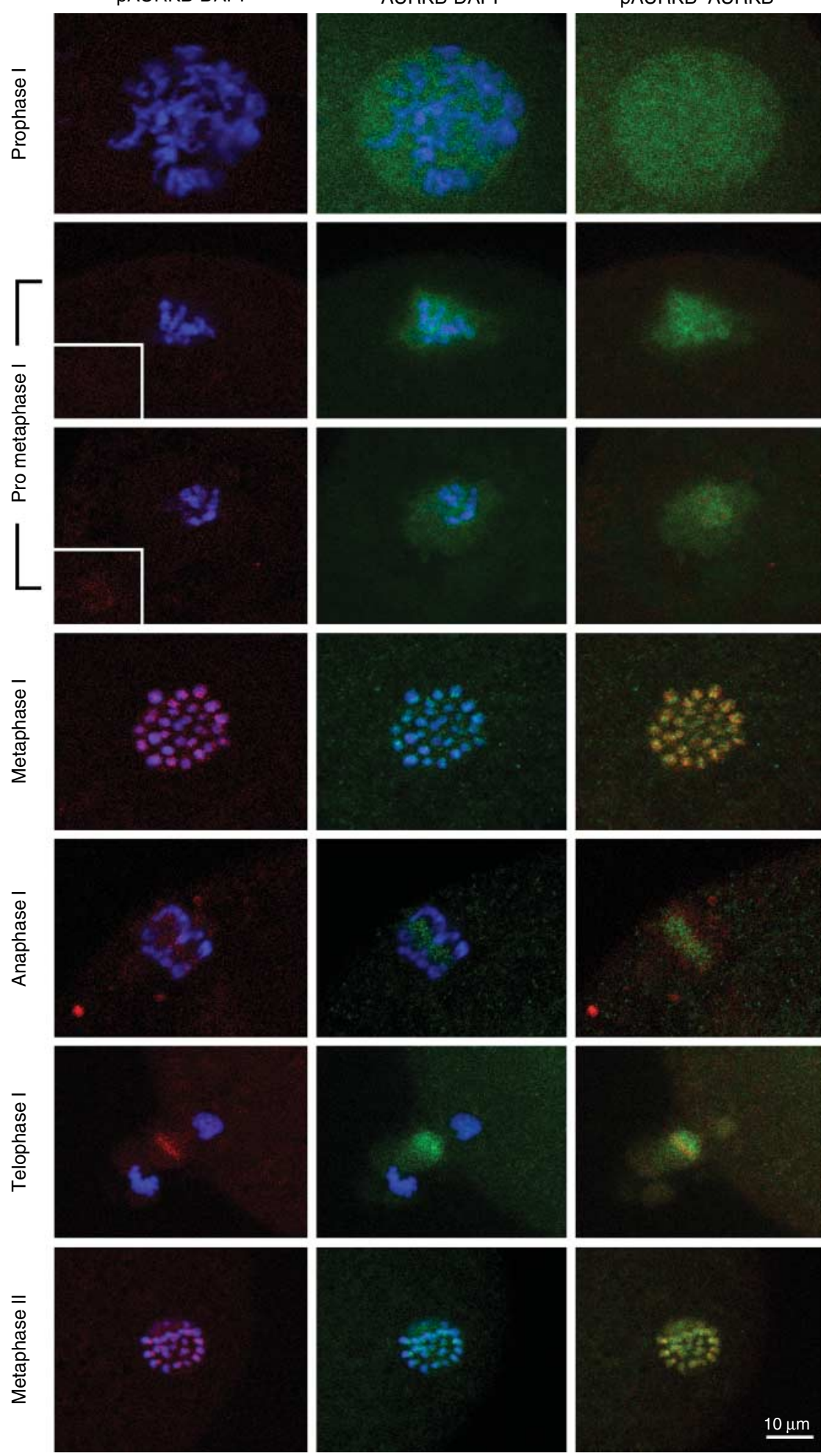

Figure 4 Colocalization of AURKB and phosphorylated AURKB during bovine oocyte maturation. Representative confocal images showing the relationship between AURKB and the active (phosphorylated) form of AURKB (pAURKB) from the prophase I (GV stage) to the methaphase II stages of meiosis. pAURKB (red) and AURKB (green) immunostaining are merged with DAPI (DNA; blue) counterstained image. In the right column, pAURKB and AURKB images are merged to reveal sites of their colocalization (orange). Images were cropped to show the area containing the chromatin. The insets in the prometaphase stage are images of pAURKB staining. This immunocytochemical analysis was conducted on a total of 239 oocytes (38 in prophase I, 18 in prometaphase I, 43 in metaphase I, 41 in anaphase I, 53 in telophase I, and 46 in metaphase II). 

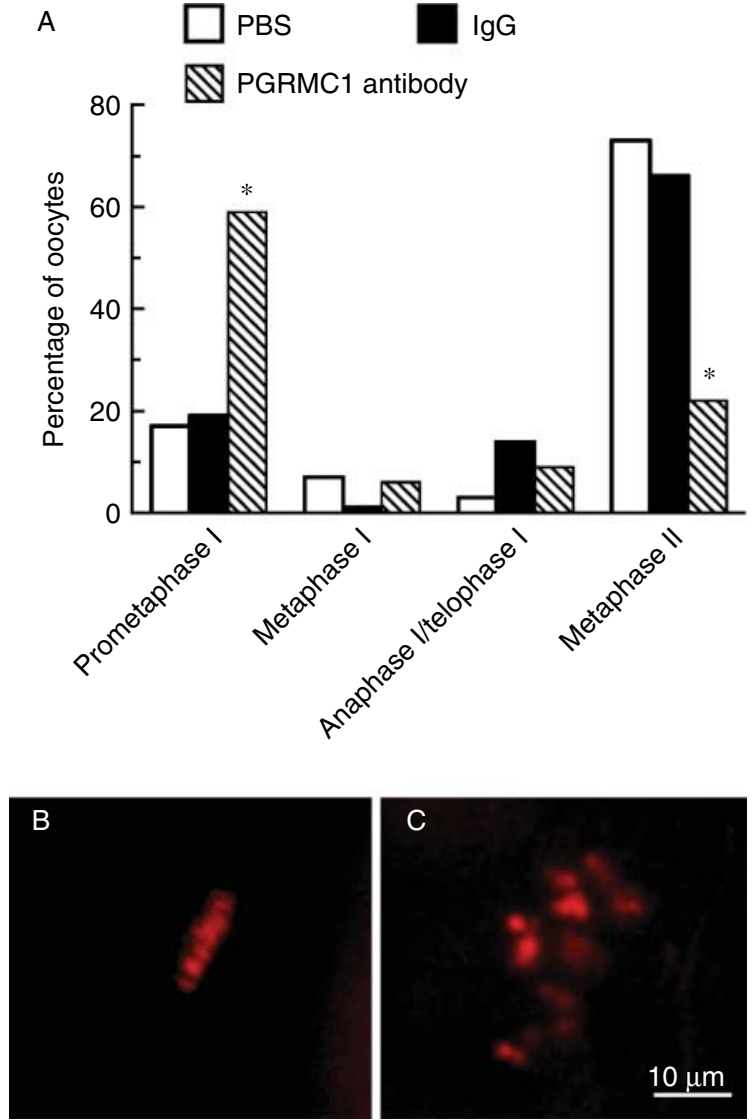

Figure 5 (A) Effect of PGRMC1 antibody injection on bovine oocyte maturation. ${ }^{*}$ Indicates a value that is significantly different $(P<0.05)$ from either PBS or IgG. After $24 \mathrm{~h}$ of culture, oocytes were stained with propididum iodide (red) to reveal the chromosomes. (B) Shows chromosomes that are precisely arranged in a metaphase plate (i.e. control) while (C) shows a bovine oocyte $24 \mathrm{~h}$ after being injected with PGRMC1 antibody. Note that the chromosomes are not aligned and disorganized.

$\approx 26 \mathrm{kDa}$ protein. This is consistent with PGRMC1 western blots obtained from lysates of other organs and cell types such as porcine liver (Meyer et al. 1998); human and rat granulosa and luteal cells (Engmann et al. 2006, Peluso et al. 2006); mouse and human smooth muscle, uterus, and placenta (Zhang et al. 2008). This study also shows that PGRMC1 is present at the GV- and MII-stage oocytes and is associated with male and female pronucleus formation of the zygote and is highly expressed in blastocysts. Specifically, before fusion of the pronuclei, PGRMC1 localizes to several foci that appear to be within nucleolar precursor bodies (NPBs; Fulka \& Fulka 2010). NPBs have been observed at the pronuclei of bovine zygotes and apparently function in nucleolus formation (Flechon \& Kopecny 1998). Moreover, NPBs are the sites of rRNA synthesis with rRNA encoding polypeptides that comprise ribosomes and therefore are essential for the synthesis of proteins that ultimately direct embryonic development (Flechon 2006).
As fertilization and embryonic development are dependent on successful oocyte maturation, the present work focused more intensely on changes in PGRMC1 after the resumption of meiosis. This study, using confocal microscopy, reveals that PGRMC1 starts to concentrate on the chromatin. This becomes more evident at metaphase I, where PGRMC1 is restricted to the centromeric region of bivalent chromatids. During anaphase I and telophase I, PGRMC1 dissociates from the chromosomes and localizes in the mid-zone and midbody respectively. At metaphase II, PGRMC1 again concentrates at the centromeric region of the chromosomes. These temporal changes in the localization of PGRMC1 during oocyte maturation suggest an important role for PGRMC1 in the process that governs meiotic progression of the oocyte.

That PGRMC1 localizing to the chromosomes is important and is supported by the finding that PGRMC1 colocalizes with AURKB at specific stages of the oocyte meiotic progression. It is well known that AURKB is expressed during oocyte meiosis in cows (Uzbekova et al. 2008), mice (Swain et al. 2008, Shuda et al. 2009, Vogt et al. 2009), and pigs (Jelinkova \& Kubelka 2006). In general, our observations on AURKB are in agreement with the known changes in the localization of AURKB, which occur during oocyte meiotic division. The one notable exception is the study by Uzbekova et al. (2008) who failed to detect AURKB within the GV, while this study did. The use of a different AURKB antibody and/or a different fixation protocol likely account for the differences observed in these two studies.

While the issue of AURKB within the GV remains to be resolved, it is important to appreciate that AURKB is a serine/threonine protein kinase, which regulates many processes during mitosis and meiosis (Kaitna et al. 2002, Shannon \& Salmon 2002, Vogt et al. 2009). In meiosis, AURKB is involved in chiasma resolution and its activation (i.e. phosphorylation on Thr232) and is correlated with cytokinesis and first polar body formation (Kaitna et al. 2002, Vogt et al. 2009). In addition, treatment with a pharmacological inhibition of AURKB results in the failure to extrude the first polar body. Furthermore, in porcine oocytes, AURKB activity increases after prometaphase I reaching a maximum at metaphase I and thereafter is maintained until metaphase II (Jelinkova \& Kubelka 2006). This study is consistent with these observations and further reveals that during telophase I AURKB localizes within the spindle midbody. Interestingly, not all of the AURKB within the spindle midbody is active. Rather only the AURKB at the center of the midbody is active (phosphorylated). Interestingly, the active fraction of AURKB appears to colocalize with PGRMC1. This implies that both PGRMC1 and AURKB are involved in the mechanism by which asymmetric cytokinesis occurs during first polar body formation. 
The fact that PGRMC1 only appears to colocalize with active AURKB raises the issue of whether PGRMC1 is essential for the activation of AURKB. Recently, a fluorescence resonance energy transfer-based assay was used to assess phospho-Thr ${ }^{232}$ AURKB activity in anaphase HeLa cells. Similar to our observations in the bovine oocycte, this study revealed that most of the AURKB activity localized to the center of the midbody (Fuller et al. 2008). These authors suggest that the spindle or spindle-associated proteins are responsible for activating AURKB. Although a previous study indicated that PGRMC1 is associated with the mitotic spindle (Nousiainen et al. 2006), the role of PGRMC1 in activating AURKB during mitosis and meiosis can be only hypothesized and considerably more studies must be conducted to resolve this issue.

In addition, PGRMC1 may be involved in meiotic events that precede polar body formation, as the present data demonstrate that PGRMC1 associates with chromatin and colocalizes with AURKB at metaphase I and metaphase II. AURKB is a component of the CPC, which in part consists of the INCENP, to which AURKB is physically associated (Ruchaud et al. 2007). Furthermore, altered AURKB expression has been suggested to increase the frequency of nondysjunction and aneuploidy in mammalian oocytes (Vogt et al. 2009) while an overexpression prevents chromosome misalignment during meiosis of mouse oocytes (Shuda et al. 2009). Collectively, these findings are consistent with the concept that the interaction between PGRMC1 and AURKB plays a role in AURKB's ability to promote homologous chromosome alignment and segregation.

This concept is supported by two observations. First, the present work demonstrates that PGRMC1 concentrates at foci on the chromatin of prometaphase l-staged oocytes and at this time, AURKB starts to be phosphorylated. As meiosis progresses, both PGRMC1 and the phosphorylated form of AURKB become concentrated on the centromeric region. Since pharmacological inhibition of AURKB activity leads to abnormal chromosome congression and alterations in the formation of the chromosomal plate (Swain et al. 2008, Vogt et al. 2009), this implicates that PGRMC1-AURKB interaction is involved in the mechanism by which chromosomal separation is mediated.

The second and probably more important observation supporting a functional role for PGRMC1 in oocyte meiotic maturation is provided by the PGRMC1 antibody injection study. In this study, PGRMC1 antibody injection at GV stage results in the improper positioning of the chromatin, affecting the ability of the chromosomes to condense properly and resolve bivalents. While these observations support a role for PGRMC1 in meiosis, they also demonstrate the need to define the exact nature of the relationship between PGRMC1 and active AURKB.
This study also supports the hypothesis that changes in the level of PGRMC1 expression or its genetic structure could predispose oocytes to undergo abnormal meiotic division, which would lead to errors in chromosome segregation and ultimately aneuploid embryos. Interestingly, there are at least two conditions in women in which PGRMC1 is altered. The first is a reduction in the level of PGRMC1 expression observed in women with an $X$; autosome translocation (i.e. $[t(X ; 11)(q 24 ; q 13)])$. This $X$ chromosome translocation likely depletes one copy of PGRMC1, thereby accounting for the reduced level of PGRMC1 in these women (Mansouri et al. 2008). The second condition involves a single base mutation in which the histidine at residue 165 is changed to arginine. Although this mutation does not change the level of PGRMC1 expression, it does reduce its functional activity by $\sim 50 \%$ (Mansouri et al. 2008). Both of these genetic alterations in PGRMC1 are detected in woman with premature ovarian failure (Mansouri et al. 2008). Whether these alterations in PGRMC1 expression or genetic structures adversely affect the functional capacity of the oocyte remain to be determined.

In summary, these data demonstrate that PGRMC1 is expressed in bovine oocytes, zygotes, and blastocysts. Our data also suggest an important role for PGRMC1 in oocyte maturation that may be specifically related to the mechanism by which chromosomes segregate and the first polar body extruded. This study also implies that PGRMC1 and AURKB interact at specific points in the meiotic cascade to ensure that errors in spindle formation, chromosome segregation, and cytokinesis do not occur. These possibilities are currently under investigation.

\section{Materials and Methods Oocyte collection and culture}

All the chemicals used in this study were purchased from Sigma Chemical Company except for those specifically mentioned. Bovine ovaries were recovered at the abattoir (IT 2270M CE, INALCA JBS S.p.A., Ospedaletto Lodigiano, LO, Italy) from pubertal females subjected to routine veterinary inspection and in accordance to the specific health requirements stated in Council Directive 89/556/ECC and subsequent modifications. Ovaries were transported to the laboratory within $2 \mathrm{~h}$ in sterile saline $(\mathrm{NaCl}, 9 \mathrm{~g} / \mathrm{l})$ maintained at $26{ }^{\circ} \mathrm{C}$. All subsequent procedures, unless differently specified, were performed at 35-38 ${ }^{\circ} \mathrm{C}$. Cumulus-oocyte complexes (COCs) were retrieved from mid-sized antral follicles $(2-6 \mathrm{~mm})$ with a 16-gauge needle mounted on an aspiration pump (COOK-IVF, Brisbane, QLD, Australia). COCs were washed in TCM-199 supplemented with HEPES buffer (20 mM HEPES, $1790 \mathrm{U} / \mathrm{l}$ heparin, and $0.4 \%$ of $\mathrm{BSA}$ ) and examined under a stereomicroscope. Only COCs medium-brown in color, with five or more complete layers of cumulus cells with oocytes with finely granulated homogenous ooplasm, were used. 
Groups of 25-30 COCs were in vitro matured in TCM-199 supplemented with $0.68 \mathrm{mM}$ L-glutamine, $25 \mathrm{mM} \mathrm{NaHCO}$, $0.4 \%$ BSA fatty acid free, $0.2 \mathrm{mM}$ sodium pyruvate, and $0.1 \mathrm{IU} / \mathrm{ml}$ of recombinant human $\mathrm{FSH}$ (Gonal-F, Serono) in humidified air under $5 \% \mathrm{CO}_{2}$ at $38.5{ }^{\circ} \mathrm{C}$ as previously described (Luciano et al. 2005). Oocytes were mechanically separated from cumulus cells either just after collection from ovaries (immature oocytes at GV stage) or after 4, 8, 12, 16, 20, and $24 \mathrm{~h}$ of culture.

\section{IVF and embryo culture}

After $24 \mathrm{~h}$ of in vitro maturation, oocytes were fertilized as previously described (Luciano et al. 2005). Briefly, the content of a straw of cryopreserved bull spermatozoa (CIZ, S Miniato, Pisa, Italy) was thawed and cells were separated on a $45-90 \%$ Percoll gradient. Sperms were counted and diluted to a final concentration of $0.5 \times 10^{6}$ spermatozoa $/ \mathrm{ml}$ in fertilization medium that was a modified Tyrode's solution (TALP) supplemented with $0.6 \%(\mathrm{w} / \mathrm{v})$ BSA fatty acid free, $10 \mu \mathrm{g} / \mathrm{ml}$ heparin, $20 \mu \mathrm{M}$ penicillamine, $1 \mu \mathrm{M}$ epinephrine, and $100 \mu \mathrm{M}$ hypotaurine. COCs and sperms were incubated for $18 \mathrm{~h}$ at $38.5{ }^{\circ} \mathrm{C}$ under $5 \% \mathrm{CO}_{2}$ in humidified air. After IVF, presumptive zygotes were washed and cumulus cells were removed by vortexing for $2 \mathrm{~min}$ in $500 \mu \mathrm{l}$ of a modified synthetic oviduct fluid (SOF; Tervit et al. 1972) supplemented with $0.3 \%(\mathrm{w} / \mathrm{v})$ BSA fraction $V$, fatty acid free, MEM essential and nonessential aminoacids, $0.72 \mathrm{mM}$ sodium pyruvate, and buffered with $10 \mathrm{mM}$ HEPES and $5 \mathrm{mM} \mathrm{NaHCO}_{3}$. Presumptive zygotes were rinsed and transferred in embryo culture medium, which was SOF buffered with $25 \mathrm{mM} \mathrm{NaHCO}$, supplemented with MEM essential and nonessential aminoacids, $0.72 \mathrm{mM}$ sodium pyruvate, $2.74 \mathrm{mM}$ myo-inositol, $0.34 \mathrm{mM}$ sodium citrate, and $5 \%$ calf serum (Gibco and Invitrogen). Incubation was performed at $38.5{ }^{\circ} \mathrm{C}$ under $5 \% \mathrm{CO}_{2}, 5 \% \mathrm{O}_{2}$, and $90 \% \mathrm{~N}_{2}$ in humidified air. Zygotes and blastocyst-stage embryos were collected after 18 and $196 \mathrm{~h}$ post insemination respectively (Lodde et al. 2009).

\section{Western blot analysis}

Freshly isolated COCs were denuded by vortexing in $500 \mu \mathrm{l}$ TCM-199 supplemented with $20 \mathrm{mM}$ HEPES and $5 \%$ calf serum (Gibco, Invitrogen, and Life Technologies) for $2 \mathrm{~min}$ at $35 \mathrm{~Hz}$. Oocytes (500-700 per extracts) and aliquots of $50 \mathrm{mg}$ ovarian cortex were homogenized in lysis buffer (10 mM Tris-Cl, pH 4, 1\% w/v NP-40, $150 \mathrm{mM} \mathrm{NaCl,} \mathrm{imM}$ EDTA, and $0.2 \%$ Zwitterion), which were supplemented with complete protease inhibitor cocktail and incubated for $1 \mathrm{~h}$ on ice then centrifuged at $14000 \mathrm{~g}$ for $15 \mathrm{~min}$. The supernatant was then mixed with $4 \times$ SDS-Laemmli loading buffer and extensively boiled for $15 \mathrm{~min}$. After centrifugation for $5 \mathrm{~min}$ at $14000 \mathrm{~g}$, each cell extract was loaded onto $12 \%$ acrylamide gel and transferred to nitrocellulose. The nitrocellulose was then incubated with $5 \%$ nonfat dry milk powder overnight at $4{ }^{\circ} \mathrm{C}$ to saturate nonspecific sites. The nitrocellulose blot was incubated with polyclonal rabbit anti-PGRMC1 (Prestige antibodies, Sigma) for $2 \mathrm{~h}$ at room temperature at a dilution of 1:500. Western blots were processed using an
HRP-conjugated anti-rabbit secondary antibody (1:2000) for $45 \mathrm{~min}$ at room temperature and developed using HRP Immuno-Blot Assay kit (Bio-Rad Laboratories).

\section{Immunofluorescence staining}

Indirect immunofluorescence staining was carried out to evaluate the cellular localization of PGRMC1, AURKB, and AURKB phosphorylated on Thr232 (phospho-Thr ${ }^{232}$ AURKB) in oocytes at different stages of meiosis (see Table 1). All the oocytes and the zygotes were freed of cumulus cells by vortexing. Then the zona pellucida was removed from the oocyte, zygotes, and embryos using $0.5 \%$ pronase as previously described (Modina et al. 2004). After being washed three times in PBS containing $0.1 \%$ polyvinylalchol (PBS-PVA), the oocytes, the zygotes, and the embryos were fixed in $4 \%$ paraformaldehyde in PBS for $15 \mathrm{~min}$ at $37^{\circ} \mathrm{C}$, and then transferred at $4{ }^{\circ} \mathrm{C}$ for the next $45 \mathrm{~min}$. The fixed oocytes, zygotes, and embryos were washed three times with PBS-PVA and permeabilized with $0.3 \%$ Triton-X 100 in PBS for $10 \mathrm{~min}$ at room temperature. Nonspecific binding was blocked by incubating the samples in $10 \%$ donkey serum and $1 \% \mathrm{BSA}$ in PBS for $30 \mathrm{~min}$ at room temperature. The samples were then incubated with the primary antibody overnight at $4{ }^{\circ} \mathrm{C}$. PGRMC1 immunolocalization was carried out using a rabbit polyclonal anti-PGRMC1 antibody (Prestige antibodies by Sigma). For the colocalization study, the oocytes were incubated either with a solution of rabbit polyclonal anti-PGRMC1 antibody and mouse monoclonal anti-AURKB antibody (Abcam, Cambridge, UK) or with a solution of mouse monoclonal anti-AURKB antibody and rabbit polyclonal anti-phospho-Thr ${ }^{232}$ AURKB antibody (BioLegend, San Diego, CA, USA). All the primary antibodies were diluted 1:50 in PBS containing 1\% BSA. After being washed three times in PBS-PVA for 10 min each, the oocytes were incubated with TRITC-labeled donkey anti-rabbit antibody (dilution 1:100; Vector Laboratories, Inc., Burlingame, CA, USA) or, for the colocalization experiment, with a solution of Alexa Fluor 488-labeled donkey anti-mouse antibody (dilution 1:500; Invitrogen and Life Technologies) and TRITC-labeled donkey anti-rabbit antibody for $30 \mathrm{~min}$ at room temperature.

The samples were washed three times in PBS-PVA and mounted on slides in the antifade medium Vecta Shield (Vector Laboratories, Inc.) supplemented with $1 \mu \mathrm{g} / \mathrm{ml}$ DAPI. Samples were analyzed on a Nikon Diaphot epifluorescence microscope or on a Nikon C1si confocal laser-scanning microscope (Nikon Corp., Tokyo, Japan). In each experiment, negative controls were performed by adding an equal concentration of rabbit IgG (Vector Laboratories) or omitting the primary antibodies and did not reveal any staining.

\section{PGRMC1 blocking antibody injection study}

The anti-PGRMC1 antibody (Prestige antibodies by Sigma) was spin dialyzed and concentrated using $10 \mathrm{kDa}$ Millipore Ultrafree 0.5 PBGC filter unit (Millipore Corp., Bedford, MA, USA). GV-stage oocytes within their cumulus cell complexes were injected with $15-35 \mathrm{pl}$ of a $1.2 \mathrm{mg} / \mathrm{ml}$ stock solution of 
anti-PGRMC1 antibody in PBS. This resulted in a final concentration of $0.15-0.30 \mu \mathrm{M}$ of antibody in the oocyte. The injected volume represented $\sim 1.5-3 \%$ of oocyte volume. As control, a group of COCs in each experiment was injected with an equal volume of rabbit IgG (Vector Laboratories), dissolved in PBS at the same concentration or PBS only. The procedure was carried out as previously described in mouse (Mehlmann et al. 2002, Jaffe \& Terasaki 2004) and adapted to bovine oocyte volume.

A microinjection apparatus (Narishige Co. Ltd, Tokyo, Japan) was used to guide the holding and injecting micropipettes into a $50 \mu \mathrm{l}$ drop of TCM-199-HEPES buffer covered with mineral oil. The injecting pipette was connected to a Femtolet (Eppendorf Srl, Milano, Italy) that allowed the injection of minute quantities of solution.

After being injected, COCs were washed in TCM-199-HEPES buffer and matured in vitro for $24 \mathrm{~h}$ as described above. After the maturation time, the oocytes were freed of cumulus cells and fixed in $500 \mu \mathrm{l}$ of $60 \%$ methanol in PBS for $30 \mathrm{~min}$ at $4{ }^{\circ} \mathrm{C}$. The oocytes were stained with $0.5 \mathrm{mg} / \mathrm{ml}$ of propidium iodide to assess the stage of meiotic progression by observation at $\times 200-400$ under fluorescence microscopy (Luciano et al. 2005).

\section{Statistical analysis}

All experiments were replicated at least three times. Observations from all the experiments were pooled. The images shown in each figure are representative. The actual number of oocytes at each stage of maturation, which were analyzed, is provided in the legend of each figure. Differences in maturation rate in the PGRMC1 blocking antibody injection study were analyzed by $\chi^{2}$-test. Probabilities of $<0.05$ were considered statistically significant.

\section{Supplementary data}

This is linked to the online version of the paper at http://dx.doi. org/10.1530/REP-10-0218.

\section{Declaration of interest}

The authors declare that there is no conflict of interest that could be perceived as prejudicing the impartiality of the research reported.

\section{Funding}

This work is supported by Programma dell'Università per la Ricerca (PUR) 2008, grant number 12-1-5284002-27 (AML), University of Milan.

\section{Acknowledgements}

The authors are grateful to Mrs Elisa Zuccari for excellent technical assistance. The authors would also like to thank Prof. Cristiano Rumio of the Department of Human Morphology, University of Milan for excellent support for LSCM.

\section{References}

Cahill MA 2007 Progesterone receptor membrane component 1: an integrative review. Journal of Steroid Biochemistry and Molecular Biology 105 16-36. (doi:10.1016/j.jsbmb.2007.02.002)

Carmena M \& Earnshaw WC 2003 The cellular geography of aurora kinases. Nature Reviews. Molecular Cell Biology 4 842-854. (doi:10. 1038/nrm1245)

Dode MA, Dufort I, Massicotte L \& Sirard MA 2006 Quantitative expression of candidate genes for developmental competence in bovine two-cell embryos. Molecular Reproduction and Development 73 288-297. (doi:10.1002/mrd.20427)

Engmann L, Losel R, Wehling M \& Peluso JJ 2006 Progesterone regulation of human granulosa/luteal cell viability by an RU486-independent mechanism. Journal of Clinical Endocrinology and Metabolism 91 4962-4968. (doi:10.1210/jc.2006-1128)

Eppig JJ, Viveiros MM, Marin-Bivens C \& De La Fuente R 2004 Regulation of mammalian oocyte maturation. In The Ovary, pp 113-129. Eds PCK Leung\& EY Adashi. Amsterdam: Elsevier/Academic Press.

Flechon JE 2006 Analysis of the nucleolar compartment of the nucleus as an indicator of nuclear reprogramming after nuclear transfer. Methods in Molecular Biology 325 225-238. (doi:10.1385/1-59745-005-7:225)

Flechon JE \& Kopecny V 1998 The nature of the 'nucleolus precursor body' in early preimplantation embryos: a review of fine-structure cytochemical, immunocytochemical and autoradiographic data related to nucleolar function. Zygote 6 183-191. (doi:10.1017/S0967199498 000112)

Fu J, Bian M, Jiang Q \& Zhang C 2007 Roles of aurora kinases in mitosis and tumorigenesis. Molecular Cancer Research 5 1-10. (doi:10.1158/ 1541-7786.MCR-06-0208)

Fulka H \& Fulka J Jr 2010 Nucleolar transplantation in oocytes and zygotes: challenges for further research. Molecular Human Reproduction 16 63-67. (doi:10.1093/molehr/gap088)

Fuller BG, Lampson MA, Foley EA, Rosasco-Nitcher S, Le KV, Tobelmann P, Brautigan DL, Stukenberg PT \& Kapoor TM 2008 Midzone activation of aurora B in anaphase produces an intracellular phosphorylation gradient. Nature 453 1132-1136. (doi:10.1038/nature06923)

Hauf S, Cole RW, LaTerra S, Zimmer C, Schnapp G, Walter R, Heckel A, van Meel J, Rieder CL \& Peters JM 2003 The small molecule Hesperadin reveals a role for aurora $\mathrm{B}$ in correcting kinetochore-microtubule attachment and in maintaining the spindle assembly checkpoint. Journal of Cell Biology 161 281-294. (doi:10.1083/jcb.200208092)

Jaffe LA \& Terasaki M 2004 Quantitative microinjection of oocytes, eggs, and embryos. Methods in Cell Biology 74 219-242.

Jelinkova L \& Kubelka M 2006 Neither aurora B activity nor histone H3 phosphorylation is essential for chromosome condensation during meiotic maturation of porcine oocytes. Biology of Reproduction $\mathbf{7 4}$ 905-912. (doi:10.1095/biolreprod.105.047886)

Kaitna S, Pasierbek P, Jantsch M, Loidl J \& Glotzer M 2002 The aurora B kinase AIR-2 regulates kinetochores during mitosis and is required for separation of homologous chromosomes during meiosis. Current Biology 12 798-812. (doi:10.1016/S0960-9822(02)00820-5)

Lodde V, Modina SC, Franciosi F, Zuccari E, Tessaro I \& Luciano AM 2009 Localization of DNA methyltransferase-1 during oocyte differentiation, in vitro maturation and early embryonic development in cow. European Journal of Histochemistry 53 199-207.

Luciano AM, Lodde V, Beretta MS, Colleoni S, Lauria A \& Modina S 2005 Developmental capability of denuded bovine oocyte in a co-culture system with intact cumulus-oocyte complexes: role of cumulus cells, cyclic adenosine $3^{\prime}, 5^{\prime}$-monophosphate, and glutathione. Molecular Reproduction and Development 71 389-397. (doi:10.1002/mrd. 20304)

Mansouri MR, Schuster J, Badhai J, Stattin EL, Losel R, Wehling M, Carlsson B, Hovatta O, Karlstrom PO, Golovleva I et al. 2008 Alterations in the expression, structure and function of progesterone receptor membrane component-1 (PGRMC1) in premature ovarian failure. Human Molecular Genetics 17 3776-3783. (doi:10.1093/hmg/ ddn274)

Mehlmann LM, Jones TL \& Jaffe LA 2002 Meiotic arrest in the mouse follicle maintained by a Gs protein in the oocyte. Science 297 1343-1345. (doi:10.1126/science.1073978) 
Meyer C, Schmid R, Schmieding K, Falkenstein E \& Wehling M 1998 Characterization of high affinity progesterone-binding membrane proteins by anti-peptide antiserum. Steroids 63 111-116. (doi:10.1016/ S0039-128X(97)00143-8)

Modina S, Beretta M, Lodde V, Lauria A \& Luciano AM 2004 Cytoplasmic changes and developmental competence of bovine oocytes cryopreserved without cumulus cells. European Journal of Histochemistry 48 337-346.

Musacchio A \& Salmon ED 2007 The spindle-assembly checkpoint in space and time. Nature Reviews. Molecular Cell Biology 8 379-393. (doi:10. 1038/nrm2163)

Nousiainen M, Sillje HH, Sauer G, Nigg EA \& Korner R 2006 Phosphoproteome analysis of the human mitotic spindle. PNAS 103 5391-5396. (doi:10.1073/pnas.0507066103)

Ota T, Suto S, Katayama H, Han ZB, Suzuki F, Maeda M, Tanino M, Terada Y \& Tatsuka M 2002 Increased mitotic phosphorylation of histone H3 attributable to AIM-1/Aurora-B overexpression contributes to chromosome number instability. Cancer Research 62 5168-5177.

Peluso J, Pappalardo A, Losel R \& Wehling M 2006 Progesterone membrane receptor component 1 expression in the immature rat ovary and its role in mediating progesterone's antiapoptotic action. Endocrinology 147 3133-3140. (doi:10.1210/en.2006-0114)

Ruchaud S, Carmena M \& Earnshaw WC 2007 Chromosomal passengers: conducting cell division. Nature Reviews. Molecular Cell Biology 8 798-812. (doi:10.1038/nrm2257)

Shannon KB \& Salmon ED 2002 Chromosome dynamics: new light on aurora B kinase function. Current Biology 12 R458-R460. (doi:10.1016/ S0960-9822(02)00945-4)

Shuda K, Schindler K, Ma J, Schultz RM \& Donovan PJ 2009 Aurora kinase B modulates chromosome alignment in mouse oocytes. Molecular Reproduction and Development 76 1094-1105. (doi:10.1002/mrd. 21075)

Sirard MA, Parrish JJ, Ware CB, Leibfried-Rutledge ML \& First NL 1988 The culture of bovine oocytes to obtain developmentally competent embryos. Biology of Reproduction 39 546-552. (doi:10.1095/biolreprod39.3.546)
Swain JE, Ding J, Wu J \& Smith GD 2008 Regulation of spindle and chromatin dynamics during early and late stages of oocyte maturation by aurora kinases. Molecular Human Reproduction 14 291-299. (doi:10. 1093/molehr/gan015)

Tervit HR, Whittingham DG \& Rowson LEA 1972 Successful culture in vitro of sheep and cattle ova. Journal of Reproduction and Fertility $\mathbf{3 0}$ 493-497. (doi:10.1530/jrf.0.0300493)

Uzbekova S, Arlot-Bonnemains Y, Dupont J, Dalbies-Tran R, Papillier P, Pennetier S, Thelie A, Perreau C, Mermillod P, Prigent C et al. 2008 Spatio-temporal expression patterns of aurora kinases $\mathrm{a}, \mathrm{B}$, and $\mathrm{C}$ and cytoplasmic polyadenylation-element-binding protein in bovine oocytes during meiotic maturation. Biology of Reproduction 78 218-233. (doi:10.1095/biolreprod.107.061036)

Vogt E, Kipp A \& Eichenlaub-Ritter U 2009 Aurora kinase B, epigenetic state of centromeric heterochromatin and chiasma resolution in oocytes. Reproductive BioMedicine Online 19 352-368. (doi:10.1016/ S1472-6483(10)60169-1)

Wang Q, Wang CM, Ai JS, Xiong B, Yin S, Hou Y, Chen DY, Schatten H \& Sun QY 2006 Histone phosphorylation and pericentromeric histone modifications in oocyte meiosis. Cell Cycle 5 1974-1982.

Wood JR, Dumesic DA, Abbott DH \& Strauss JF III 2007 Molecular abnormalities in oocytes from women with polycystic ovary syndrome revealed by microarray analysis. Journal of Clinical Endocrinology and Metabolism 92 705-713. (doi:10.1210/jc.2006-2123)

Zhang L, Kanda Y, Roberts DJ, Ecker JL, Losel R, Wehling M, Peluso JJ \& Pru JK 2008 Expression of progesterone receptor membrane component 1 and its partner serpine $1 \mathrm{mRNA}$ binding protein in uterine and placental tissues of the mouse and human. Molecular and Cellular Endocrinology 287 81-89. (doi:10.1016/j.mce.2008.02.012)

Received 11 May 2010

First decision 23 June 2010

Revised manuscript received 17 August 2010

Accepted 25 August 2010 